polyarthritis, the lower limbs being predominantly affected, knees and ankles most commonly but fingers, toes, and wrists also, and occasionally the lumbar spine, hips, shoulders, jaw or sacroiliac joints, usually in an asymmetrical pattern. The joints are tender and swollen and effusions are not uncommon.

Until recently reports of such infections were mostly from Scandinavia and Central Europe, but Thomas, Solomon, and Rabson ${ }^{4}$ state that over 100 cases have now been diagnosed at the South African Institute for Medical Research. Erythema nodosum, a not uncommon manifestation of the disease in other countries, ${ }^{5}$ is a relatively rare finding in South Africa, but a septicaemic form of the disease with a $50 \%$ mortality rate is there relatively more common than elsewhere, ${ }^{6}$ particularly in patients with hepatic cirrhosis or iron overload. The department of orthopaedic surgery in Witwatersrand University, Johannesburg has recently seen ${ }^{4} 11$ patients with infection attributed to $Y$. enterocolitica, 10 of whom presented with a polyarthritis and one with a backache. Of the ten patients with polyarthritis four had also an associated backache. Five were women, six men, ages ranging from 18 to 51 years. Abdominal pain or diarrhoea was present in five and absent in six. Sedimentation rates were raised in all but two patients; a leucocytosis was present in only four. Most were febrile. Stool cultures were negative, and the diagnosis was made on $\mathrm{H}$ agglutination tests, titres varying from $1 / 100$ to $1 / 6400$. Blood cultures were negative in all cases. The polyarthritis tended to be flitting in nature, and though never disappearing from any joint fluctuated in intensity from one area to another. Some joints were hot, red, swollen and tender resembling an acute pyarthrosis, some were less acute, resembling rheumatoid arthritis. Articular erosions were seen in the $x$-ray films of only one patient, who had sacroiliitis. Treatment was essentially symptomatic and somewhat unsatisfactory, though combined antibiotic and anti-inflammatory therapy was associated with improvement in 10 of the 11 patients. But, as the authors state, this improvement may well have been due to the natural regression of the disorder.

This clinical picture of the arthropathy is very similar to that previously described by workers in Finland, ${ }^{7}$ who found the duration of acute joint symptoms ranged usually from one to four months, fluid aspirated from the affected joints showing usually a polymorphonuclear pleocytosis. One point of difference is that though the Finnish workers noted slight radiological changes suggesting a mild left sacroiliitis in one case there is no mention of backache in the nine patients they reported. On the other hand they noted signs of carditis in 6 patients, whereas no mention is made of cardiac findings in the South African patients. These differences may well have been due to differences of observation and emphasis. In view, however, of the importance of the histocompatability antigen HL-A 27 (W 27) and its relation to disease, particularly the inflammatory spondylarthropathies, cases such as those reported from South Africa should be tissue-typed and followed for several years. Both in Britain ${ }^{8}$ and the U.S.A. ${ }^{9}$ patients with ankylosing spondylitis have been found in a large majority of cases to have the HL-A 27 (W 27) antigen, and this has also been shown to be true of spondylitis secondary to Reiter's disease, ${ }^{1012}$ psoriasis, ${ }^{11} 12$ the enteropathic arthropathies associated with ulcerative colitis and Crohn's disease, ${ }^{1213}$ and some male cases of juvenile rheumatoid arthritis. ${ }^{14}$ Indeed, it has been suggested ${ }^{15}$ that these disorders be called collectively "W27 rheumatic disease." Of particular interest in relation to yersinia infection are the publications by Professor Aho and his colleagues in Helsinki. ${ }^{16-18}$ They found the histocompatability antigen HL-A 27 in 43 of 49 patients with yersinia arthritis and 36 of 40 patients with Reiter's disease, compared with 3 of 20 patients with yersinia infection without arthritis and $14 \%$ of the normal Finnish population. They observe that instead of an orientation towards individual clinical syndromes there is now more emphasis "on the role of a host genetic factor in the mediation of common pathologic expressions of multiple aetiologic stimuli." The old saying that the soil is as important as the seed in development of disease would seem to be as true in rheumatology as in the rest of medicine.

1 Ahvonen, P., Sievers, K., and Aho, K., Acta Rheumatologica Scandinavica $1969,15,232$.

2 Hällström, K., Sairanen, E., and Ohela, K., Acta Medica Scandinavica, $1972,191,485$.

4 Thomas A. F., Solomon, L., and Rabson, A., South African Medical fcurnal, 1975 49, 18.

5 Mygina, N., and Thulin, H., British fournal of Dermatology, 1970, 82, 351.

- Mollaret, H. H., Pathologie et Biologie, 1971, 19, 189.

Laitinen, O., Tuuhea, J., and Ahvonen, P., Annals of Rheumatic Disease, 1972, 31, 34.

8 Brewerton, D. A., et al., Lancet, 1973, 1904.

9 Schlosstein, L., et al., New England fournal of Medicine, 1973, 288704.

10 Brewerton, D. A., $t$ al., Lancel, 1973, 2, 996

11 Brewerton, D. A., et al., Lancet, 1974 1, 956.

12 McClusky, O. E., Lordon, R. E., and Arnett, F. C., fournal of Rheumatology, 1974 1, 263.

13 Russell, A. S., et al., fournal of Rheumatology, 1974, 1, 203.
14 Sturrock, R. D., et al., fournal of Rheumatology, 1974, 1, 269.

15 Metzger, A. L., Morris, R. I., and Bluestone, R., fournal of Rheumatology, $1974,1,274$.

${ }_{17}$ Aho, K., et al., Lancet, 1973, 2, 157.

17 Aho, K., et al., Arthritis and Rheumatism, 1974, 17, 521.

18 Aho, K., Annals of Rheumatic Disease, 1975. In press.

\section{How Innocuous is the White Medicine?}

Everyone is now aware that one drug may change the clinical effect of another when they are taken together, but some commonplace remedies have not been questioned because they seem to be harmless. Antacid preparations have seemed innocent in this respect, and this partly stems from some uncertainty about their efficacy in treating peptic ulcer and in neutralizing gastric acidity. They are, however, less innocuous than was thought; for it seems that some can alter drug elimination from the body by changing urinary acidity. ${ }^{1}$

The kidney is the most important route for the excretion of some drugs, and it is ideally suited for this role because it is an efficient filter, passing through the glomerulus a large volume of fluid and with it those molecules small enough to pass through the capillary pores. Most drug molecules are sufficiently small, and the fraction not bound to plasma proteins will come through into the filtrate. However, the tubular epithelium reabsorbs water and solutes, including drugs, and as with other membranes this may be an active or passive process. Furthermore some drugs, such as penicillin, are secreted into the tubular fluid. Non-ionized drug molecules cross membranes easily, but not ionized ones, and the dissociation constant ( $\mathrm{pK}_{\mathrm{a}}$ value) of the drug itself determines in part the degree of ionization. The other factor is the $\mathrm{pH}$ of the solvent; in this instance, the tubular fluid. Most drugs contain weak acidic and basic groups, and if the tubular fluid is acidic there will be more ionization of basic drugs, which will not be reabsorbed so readily, and their renal elimination will be enhanced. Alteration of $\mathrm{pH}$ towards alkalinity will have the opposite effect. The converse happens with acidic ones. 
This partly explains why alkaline diuresis promotes the excretion of more acidic drugs, such as salicylates, by stopping back-diffusion through the tubular epithelium. The other property of each drug which also plays an important part in membranediffusion is lipid solubility, and this can vary between two drugs with identical $\mathrm{pK}_{\mathrm{a}}$ values (e.g., salicylic acid and penicillin); penicillin'is poorly lipid-soluble and not reabsorbed.

The next relevant fact in any consideration of renal elimination of drugs is the consistency of urinary $\mathrm{pH}$ under normal conditions. In fact the $\mathrm{pH}$ of the excreted urine is fairly constant in any one individual, but a wider range $(\mathrm{pH} 4 \cdot 5-8 \cdot 0)$ is encountered in the population as a whole. There is a diurnal rhythm, and a high protein diet increases acidity. Against this background we can judge the likely effects of drugs on urinary $\mathrm{pH}$. Several antacid preparations decrease urinary acidity: some more than others and some not at all. In a recent study ${ }^{1}$ the most marked effects were seen with an aluminium and magnesium hydroxide suspension (mean change 0.9 ), and there were moderate changes $(0.4$ to 0.5$)$ with calcium carbonate-glycine suspension and magnesium hydroxide. Other products tested (aluminium hydroxide and dihydroxyaluminium aminoacetate) had no real effect. All were taken according to the recommended dosage for a week, and the changes in urinary $\mathrm{pH}$ were achieved within three days and remained constant thereafter.

These alkaluric effects were greatest in those individuals who had a more acidic urine in the pretreatment period; increases of more than 1.0 were recorded. With changes of this magnitude the gradient of diffusible (non-ionized) molecules of an acidic drug would increase between the fluid in the distal

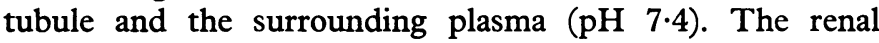
elimination of acidic drugs would be raised, but basic drugs would be reabsorbed more readily. The clinical effect would also depend on the fraction of unchanged, active drug normally eliminated through the kidneys. Specific effects have not been studied in detail, but it seems likely that the acidic drugs whose plasma levels might be decreased after antacid medication might include phenobarbitone, salicylic acid, and several sulphonamides; basic drugs which normally have a high urinary excretion and also have an appropriate dissociation constant include amphetamine, procaine amide, quinidine, ephedrine, amantadine, and salbutamol. In fact, urinary $\mathrm{pH}$ changes have already been linked to intoxication with quinidine and E.C.G. changes, ${ }^{2}$ and the plasma steady-state level of salicylic acid was halved with alkalinization of the urine. ${ }^{3}$ Clinical effects have been detected when looked for in patients on these categories of drugs.

There is a real chance that patients taking antacids might experience an unexpected alteration in urinary excretion of other drugs. Antacids not only alter the renal excretion of some drugs, they also have effects on the intestinal absorption. They can increase the absorption of bishydroxycoumarin, though not warfarin, probably by forming a more soluble complex, ${ }^{4}$ but conversely they chelate with tetracyclines forming less soluble complexes. ${ }^{5}$ Unfortunately, when asked about any drugs they might be taking, most patients tend to forget about antacids, often because they have obtained them without prescription. Doctors cannot afford to ignore "the white medicine" in their questioning about drug ingestion, for it may be less innocuous than we had assumed.

\footnotetext{
1 Gilbaldi, M., Grundhofer, B., and Levy, G., Clinical Pharmacology and

Therapeutics, 1974, 16, 520.
Gerhardt, R. E., et al., Annals of Internal Medicine, 1969, 71, 927.

2 Gerhardt, R. E., et al., Annals of Internal Medicine, 1969, 71, 927. 1971, 217,81 .

Ambre, J. J., and Fischer, L. J., Clinical Pharmacology and Therapeutics, $1973,14,231$.
}

\section{Disaster Planning-Fact or Fiction?}

Since 1954 National Health Service hospitals have been required $^{1}$ to make arrangements for dealing with casualties resulting from a major accident, but unlike hospitals in the U.S.A. there has been no obligation for these plans to be tested to see if they actually work. Publications on disaster planning were already numerous ${ }^{2-8}$ at the time of our last leading article, ${ }^{9}$ and the eruption of urban guerilla warfare in the United Kingdom has provided an opportunity of studying the plans of a number of hospitals in action. ${ }^{10-12}$ Other disasters, including the Staines plane crash, the Summerland fire, ${ }^{13}$ the Flixborough explosion, and the Moorgate train crash, have produced different problems in disaster management, but not one of them produced a new problem. Renewed interest in the practical aspects of disaster planning has been reflected in a number of seminars and conferences on the subject, ${ }^{14} 15$ but it seems that all the problems and techniques of disaster management have not as yet been appreciated, let alone put into practice, by those responsible in the hospital service and the community.

By definition, disasters occur with little or no warning, ${ }^{8}$ and casualties may start arriving at the hospital before the official alert has been given. Authority to initiate a hospital's disaster plan must be given to a doctor, nurse, or administrator actually in the hospital building at the time. Experience in Belfast confirms that adequate medical care in a disaster can often be provided by the on-duty medical and surgical teams, and that, by using a phased response, the alerting of medical, nursing, administrative, and ancillary staff can be adjusted to the nature of the disaster. ${ }^{11}$ Hospital staff should be alerted by a detailed fan-out notification system to prevent the hospital switchboard from being overloaded with routine calls, ${ }^{15}$ and staff should be allocated to their posts by the use of action cards kept on permanent display. ${ }^{16} 17$ Both the fan-out notification system and the action cards need to be tested regularly and relevant details of their role in the disaster plan should be explained to all new members of staff as part of their induction programme. The key to control of a sudden casualty load is a well-developed and tested casualty management plan. All casualties should be identified by a unique number and then undergo triage-sorting into categories of those needing urgent and non-urgent treatment, and those that are dead. Standard hospital documentation should be used wherever possible. One of the basic fundamentals of disaster planning is that wherever possible individuals should perform familiar tasks with familiar equipment in familiar places.

The concept of one "designated" hospital enshrined in H.M.(54)51 remains a stumbling block to present-day disaster planning on a district, area, or regional basis. Spreading the casualty load in distance and time to a number of casualty receiving hospitals is the key to preventing one particular hospital from being swamped with casualties, and responsibility for this rests ultimately with the ambulance service. The ambulance service remains the Cinderella of the emergency services in both status and finance, but it is becoming increasingly recognized that the ambulance man has a vital part to play in primary medical care. The number of areas with advanced training schemes for ambulance men is increasing, and the division of the service into two tiers with separation of the patient-care from the transport function cannot long be delayed. Experienced medical staff are important at the scene of an accident or disaster, but a well trained, equipped, and 